\title{
STAR FORMATION IN THREE NEARBY CLOUD COMPLEXES
}

\author{
NEAL J. EVANS II \\ Astronomy Department, \\ The University of Texas, Austin Texas 78712, USA \\ E-mail NJE@ASTRO.AS.UTEXAS.EDU \\ ELIZABETH A. LADA \\ Harvard-Smithsonian Center for Astrophysics, \\ 60 Garden Street, Cambridge, MA 02138, USA \\ E-mail LADA@CFA.BITNET
}

\begin{abstract}
We consider the distribution and nature of the gas and star formation in three nearby molecular cloud complexes : Taurus-Auriga, Ophiuchus, and Orion. Large-scale studies show that quite different distributions of dense gas and star formation exist in these regions, but that the global efficiency of star formation, so far, is about $1 \%$ in all of these complexes, very similar to the final efficiencies deduced for open clusters and associations. Furthermore, evidence for differences in the initial mass function among these regions is weak; all three regions may be compatible with the initial mass function for open clusters and field stars. Finally, we consider specific examples of star formation in each complex; the results indicate that the ideas of Adams, Lada, and Shu (1987) are reasonably consistent with the data on L1551 IRS 5, in the Taurus cloud, but that modifications are likely to be needed for IRAS 16293-2422 in Ophiuchus and for NGC2071 in Orion.
\end{abstract}

Keywords : star formation, molecular clouds, embedded clusters

\section{Introduction}

A major goal of star formation studies is to understand the physical processes by which stars originate. Consequently, current research has focused on determining the relationship between young stars and the physical and environmental conditions in the regions where they form. Two different approaches have been used to study this problem in .our Galaxy. One approach involves investigations of the processes of star formation on global scales, while another approach concentrates on local processes, by studying individual sites of star formation. Both approaches seek to compare the raw materials with the recent products of star formation.

Global studies compare the large-scale distribution and properties of the gas and dust with those of young stellar objects (YSOs). Such knowled re helps us to determine what conditions are necessary for star formation and whetl er the nature of the star forming process varies with physical conditions or the environment of the cloud. For example, clouds with differing internal or environmental conditions may form stars with a different efficiency, or with a different initial mass function. Such differences would provide insights into the connection between the raw materials and the finished products.

On a local scale, we again compare the physical conditions with the nature of the objects. We are particularly interested in understanding the formation and very 
early evolution of individual objects; this interest launched the crusade to detect protostars (e.g., Wynn-Williams 1982), generally characterized by infalling gas. The meager results of this crusade have led to suggestions that infall and outflow may occur simultaneously, and that infall may actually be intimately involved in the production of the ubiquitous outflows (Shu 1991), thus cleverly masking its own presence. Models of this type have made detailed predictions for the density distributions around individual forming stars (Adams, Lada, and Shu 1987). Observational studies of the nature of such objects allow us to test these predictions.

Nearby molecular cloud complexes provide excellent laboratories for investigations of the star forming process. Such regions have the advantage that they can be studied with high angular resolution and high sensitivity. There are several such complexes within about $150 \mathrm{pc}$, such as the Taurus-Auriga complex and the Ophiuchus complex, which are forming stars of moderate mass, but the nearest complex which is forming a significant number of truly massive stars is the. Orion complex, at a distance of about 400 pc.

Both the Taurus-Auriga region and the Orion complex have been the subject of extensive optical study, including surveys for stars with $\mathrm{H} \alpha$ emission (see Herbig and Rao 1972 for a compilation) and follow-up spectroscopy (Cohen and Kuhi 1979). Comparing the results for these two clouds, Larson (1982) noticed some apparent differences between star formation in these clouds. In Taurus, where the gas is "relatively dispersed", the clouds are forming "scattered small groups" of stars, "predominantly of low mass". In contrast, in the Orion region, "massive condensed clouds", are forming stars "mostly in a single large cluster" which is "relatively depleted in low-mass stars". A comparison of the median mass of the T Tauri stars in the two clouds indicated a value about twice as high in Orion as in Taurus (1.1 $\mathrm{M}_{\odot}$ versus $0.6 \mathrm{M}_{\odot}$ ), according to Larson (1986). No such conclusions could be drawn for Ophiuchus based on optical data because few stars were visible optically. Later infrared studies of Ophiuchus revealed many more stars, raising the question of whether similar studies of the other regions would change the conclusions based on optical studies.

In this paper, we will review recent observational results from both global and local studies of the star-forming processes in three nearby molecular cloud complexes : Taurus-Auriga, Ophiuchus, and Orion. In the next section, we compare the distributions of dense gas and stars; in section III, we compare rough estimates of the star formation efficiency and initial mass function in the three regions. Section IV contains a summary of detailed studies of a particular region of star formation in each complex.

\section{Distribution of Gas and Star Formation}

\section{1. TAURUS-AURIGA MOLECULAR CLOUD COMPLEX}

The Taurus-Auriga complex is one of the best studied examples of a nearby star forming region. It is located at $\sim 140 \mathrm{pc}$ (Elias 1978a) and contains $\sim 10^{4} \mathrm{M}_{\odot}$ of 
material (Wouterloot and Habing 1985; Cernicharo, Bachiller and Duvert 1985; Ungerechts and Thaddeus 1987). Studies of the large scale structure of TaurusAuriga, using ${ }^{13} \mathrm{CO}$ (Kleiner and Dickman 1984, 1985), star counts (Cernicharo, Bachiller and Duvert 1985), OH (Wouterlout and Habing 1985), CO (Ungerechts and Thaddeus 1987) and IRAS $100 \mu \mathrm{m}$ optical depth (Scalo 1990), have revealed complex, irregular, and filamentary structures.

In addition to the diffuse, filamentary structure, small dense cores have been found in Taurus-Auriga by molecular line surveys of regions of optical obscuration (Myers, Linke, and Benson 1983; Myers and Benson 1983). The properties of these cores have been determined from observations of the $\mathrm{NH}_{3}$ molecule, which is sensitive to gas densities $\geq 10^{4} \mathrm{~cm}^{-3}$ (Myers and Benson 1983; Benson and Myers 1989). These observations reveal that the cores are small, having sizes on the order of $0.1 \mathrm{pc}$ and masses on the order of a few $\mathrm{M}_{\odot}$. In addition the cores are cold $\left(\mathrm{T}_{\text {kinetic }} \sim 10 \mathrm{~K}\right)$ and the $\mathrm{NH}_{3}$ lines have very narrow, nearly thermal, linewidths (FWHM $\sim 0.3 \mathrm{~km} \mathrm{~s}^{-1}$ ). However, the linewidths of other molecules are clearly not thermal (Zhou et al. 1989). One should note that these properties are not restricted to Taurus cores but are typical of most dense cores found in nearby dark clouds (Myers and Benson 1983; Benson and Myers 1989).

One of the most notable characteristics of nearby dense cores is their association with star formation. Many dense cores are associated with known T Tauri stars (Myers and Benson 1983), bipolar outflows (Fuller and Myers 1987; Myers et al. 1988) and low luminosity IRAS sources (Beichman et al. 1986; Myers et al. 1987). In fact, IRAS sources have been detected in approximately half of the $\sim 100$ cores which are known in Taurus and other nearby complexes (Benson and Myers 1989). Typically, one to a few stars are found in a core.

An understanding of the global processes of star formation in a given molecular cloud complex requires knowledge of the distribution of young stellar objects within that complex. The first large-scale $\left(120 \mathrm{pc}^{2}\right)$ infrared survey of the Taurus region was obtained at $2 \mu \mathrm{m}$ (Elias 1978a) with a single detector. Consequently the spatial resolution was poor $\left(1^{\prime}-2^{\prime}\right)$ and only a modest number $\left(1.8 \times 10^{4}\right)$ of "pixels" could be obtained. In addition, sensitivity was poor, with a limiting magnitude of only $\mathrm{m}_{K}=7.5$. Nonetheless, Elias was able to find 215 sources, most of which he concluded were field stars. The stars which were associated with the complex appeared to be in small groups, scattered through the cloud, in agreement with conclusions based on optical studies (Cohen and Kuhi 1979; Larson 1982).

Recently, Kenyon et al. (1990) have completed a survey of the embedded stellar population in the Taurus molecular cloud, using the IRAS data base, followed by near-infrared and optical studies. Their survey is complete for luminosities $\mathrm{L}>$ $0.5 \mathrm{~L}_{\odot}$, making it one of the most sensitive surveys of an embedded population in a large complex. They have identified at least seven new pre-main-sequence stars and six new deeply embedded objects. The distribution of the young stellar objects within the Taurus region is shown in Figure 1, superimposed on a map of CO integrated intensity (from Kenyon et al. 1990). Roughly 100 objects are located 
within a $40 \mathrm{pc} \times 30 \mathrm{pc}$ area. Although some concentrations are seen in this figure, the sources for the most part appear scattered throughout the cloud, in agreement with the early findings.

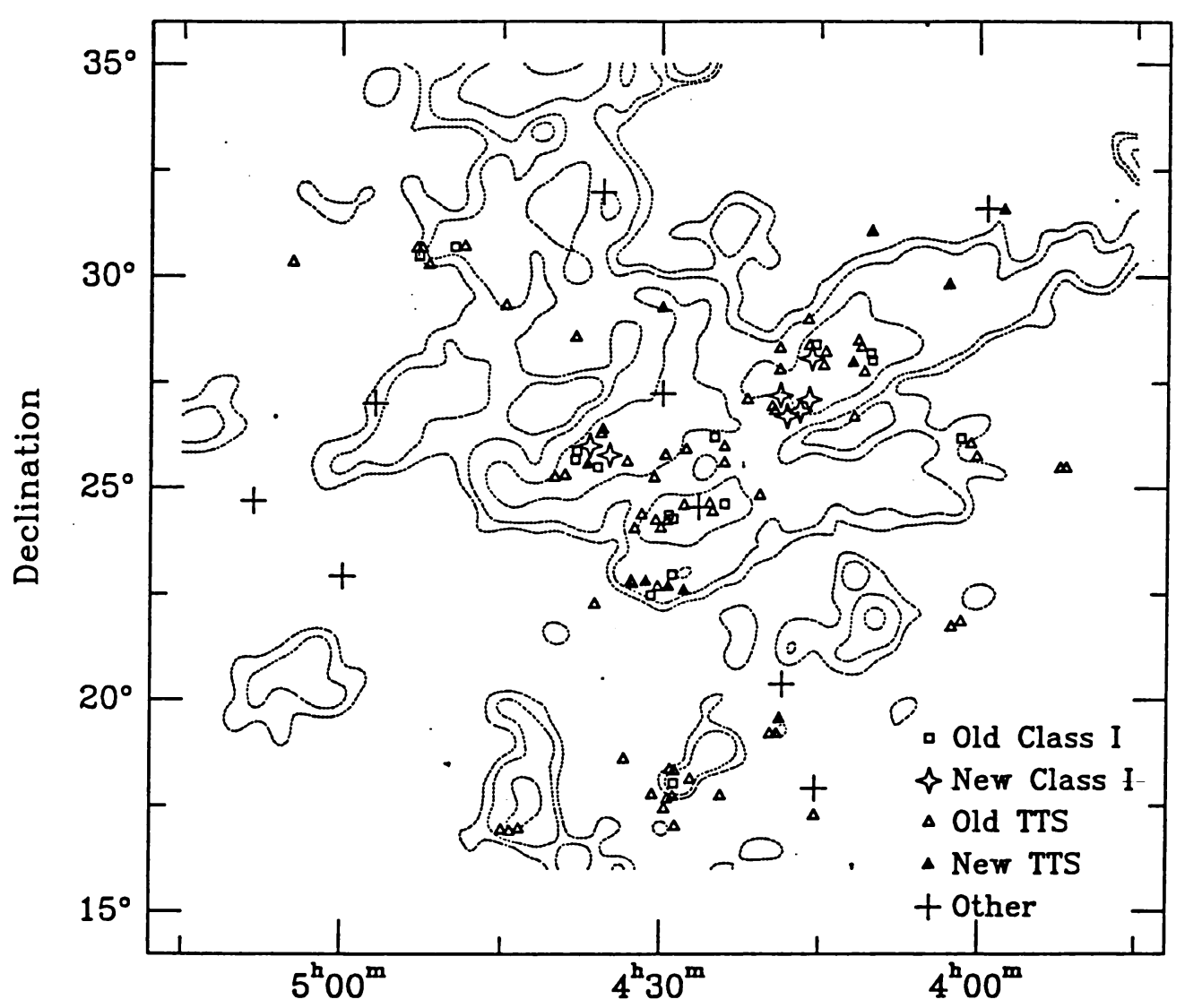

Right Ascension

Fig. 1. Distribution of young stellar objects in the Taurus-Auriga Molecular Cloud Complex (from Kenyon et al. 1990). The distribution of YSOs is shown superimposed on a map of $C O$ integrated intensity (Ungerechts and Thaddeus 1987). The CO contours are presented for integrated intensities equal to $3,5,10,25$ and $40 \mathrm{~K}$ $\mathrm{km} \mathrm{s}^{-1}$. 


\section{2. OPHIUCHUS MOLECULAR CLOUD COMPLEX}

The Ophiuchus molecular cloud complex (see Wilking 1990 for a review) is another well studied nearby region of star formation, located near the Scorpius-Centaurus OB association. Distance estimates to the molecular complex range from the traditional value of $160 \mathrm{pc}$ (Bertiau 1958, Whittet 1974, and Chini 1981) to a more recent value of $125 \pm 25 \mathrm{pc}$ by de Geus et al. (1989), who argue that the near side of the cloud is as close as $80 \mathrm{pc}$, while the back side is at $170 \mathrm{pc}$. Many similarities exist between the Ophiuchus and Taurus complexes. The total mass of the Ophiuchus complex, measured in $\mathrm{CO}$, is also about $10^{4} \mathrm{M}_{\odot}$ (de Geus, Bronfman and Thaddeus 1990). Large scale molecular line surveys in CO (de Geus, Bronfman and Thaddeus 1990) and ${ }^{13} \mathrm{CO}$ (Loren 1989a, b) have revealed filamentary or clumpy structures. In addition to the 89 distinct ${ }^{13} \mathrm{CO}$ structures identified by Loren $(1989 \mathrm{a}, \mathrm{b})$, several dense $\mathrm{NH}_{3}$ cores, with properties similar to those found in Taurus, have been identified in this complex (Myers and Benson 1983; Benson and Myers 1989).

The Ophiuchus star forming region is distinguished from Taurus by the presence of a large centrally condensed core (Wilking and Lada 1983), located in the $\rho$ Ophiuchi cloud, the westernmost cloud of the complex (see Klose 1986 for a review). The core is heavily obscured, with $\mathrm{A}_{v} \sim 100 \mathrm{mag}$ (Vrba et al. 1975; Wilking and Lada 1983). $\mathrm{C}^{18} \mathrm{O}$ observations indicate a core $1 \mathrm{pc} \times 2 \mathrm{pc}$ in size, containing $\sim 600 \mathrm{M}_{\odot}$ (Wilking and Lada 1983). There are also concentrations of dense ( $\mathrm{n} \geq$ $10^{4} \mathrm{~cm}^{-3}$ ) gas in the core, as indicated by observations of $\mathrm{H}_{2} \mathrm{CO}$ (Loren, Sandqvist and Wootten 1983) and $\mathrm{DCO}^{+}$(Loren, Wootten and Wilking 1990).

The Ophiuchus Complex is further distinguished from the Taurus Complex by the localized concentration of young stars in the $\rho$ Ophiuchi cloud. $\mathrm{H} \alpha$ objective prism surveys of the Ophiuchus complex (Struve and Rudkjobing 1949; Haro 1949; Dolidze and Arakelyan 1959; Wilking, Schwartz and Blackwell 1987) have shown that the majority of young stars are associated with the western half of the $\rho$ Ophiuchi cloud. In addition to optical studies, a large area $\left(140 \mathrm{pc}^{2}\right)$ in this complex was surveyed at $2.2 \mu \mathrm{m}$ by Elias (1978b), with similar sensitivity, resolution, and number of pixels to those obtained in his study of Taurus-Auriga (Elias 1978a). Elias found nearly twice as many sources per unit area in Ophiuchus, though again most were field stars. In contrast to the distribution in Taurus, the associated sources in Ophiuchus were concentrated into a single group, later associated with the region of high molecular column density in the $\rho$ Ophiuchi cloud (Wilking and Lada 1983). More recently, IRAS based studies of the embedded stellar population in Ophiuchus (Ichikawa and Nishida 1989) have also revealed a concentration of IRAS sources in the $\rho$ Ophiuchi cloud. These results confirmed the earlier suggestions of the presence of a dense stellar cluster in the $\rho$ Ophiuchi cloud (Grasdalen, Strom, and Strom 1973; Vrba et al. 1975).

The embedded stellar cluster in the $\rho$ Ophiuchi core has been studied in detail using both near-infrared and IRAS observations (Wilking and Lada 1983; Lada and Wilking 1984; Young, Lada and Wilking 1986; Wilking, Lada and Young 1989). 
The distribution of embedded sources is shown in Figure 2. A total of 78 objects have been identified as members of the cluster, and 39 additional infrared sources lie within the core boundary but remain unclassified (Wilking, Lada and Young 1989). Therefore, roughly 100 embedded young stellar objects are located within the $1 \mathrm{pc} \times 2 \mathrm{pc}$ area of the core.

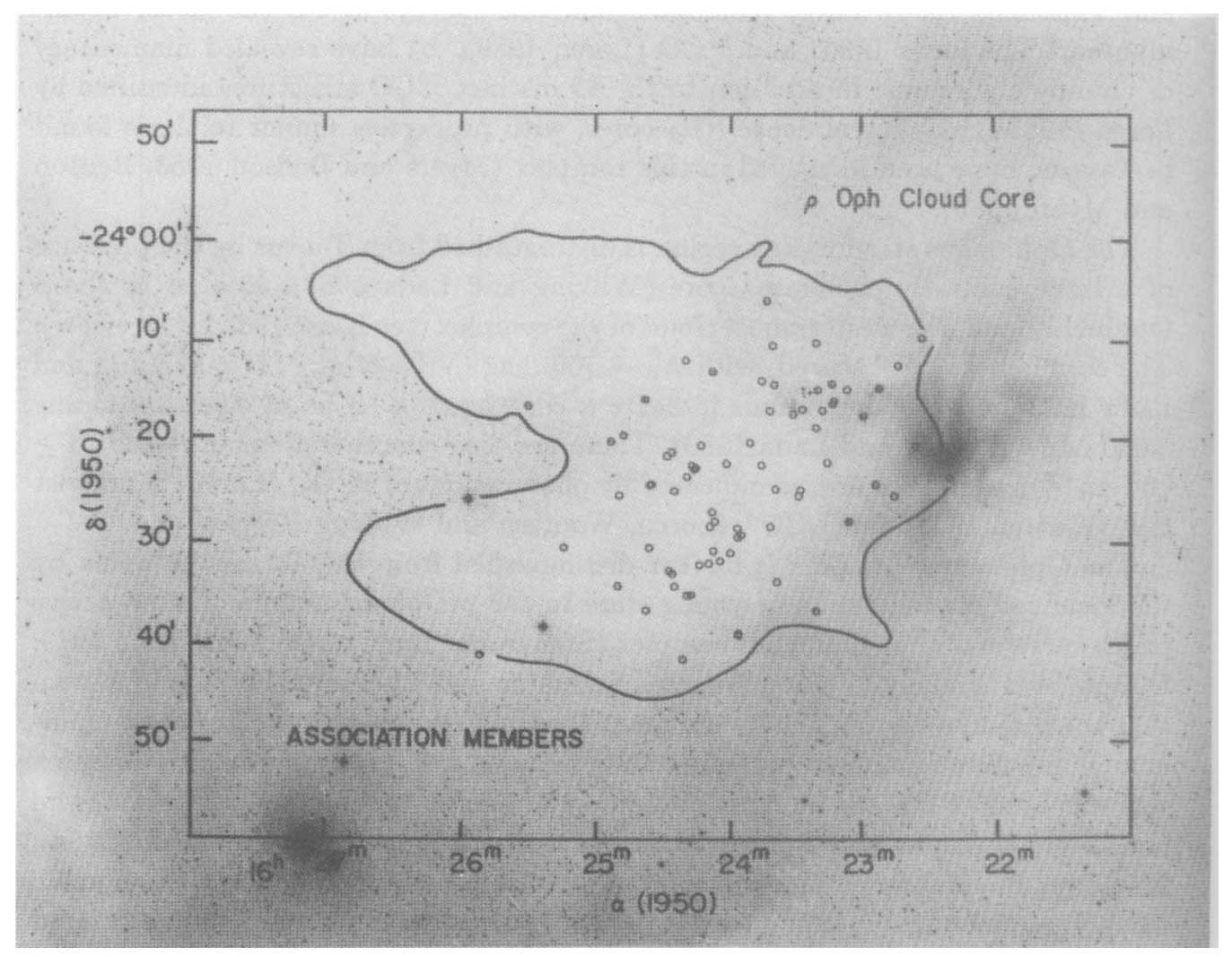

Fig. 2. The distribution of young stellar objects associated with the $\rho$ Ophiuchi Molecular cloud (from Wilking, Lada and Young 1989). YSOs associated with the molecular cloud core are displayed as open circles, superposed on the red photograph of the Palomar Sky Survey. The boundaries of the molecular gas $\left({ }^{3} \mathrm{CO}\right.$, Loren 1989a) are presented by a solid contour. 


\section{3. THE ORION MOLECULAR CLOUDS - L 1641 AND L 1630}

The nearest region of copious production of massive stars is in the direction of Orion. This complex is usually assumed to lie at a distance of $500 \mathrm{pc}$, but an extensive study of the distances to B stars indicates a distance of about $400 \mathrm{pc}$ (Anthony-Twarog 1982); this estimate is consistent with the low end of the range $(480 \pm 80 \mathrm{pc})$, based on proper motions of the $\mathrm{H}_{2} \mathrm{O}$ masers in the $\mathrm{OMC1}$ region (Genzel et al. 1981), but the uncertainties are considerably less. A large-scale map of the region in CO emission (Maddalena et al. 1986) reveals two major clouds : Orion A (L1641), with a mass of $1.0 \times 10^{5} \mathrm{M}_{\odot}$, including the Orion Nebula region and extending southward; and Orion B (L1630), extending northward from the Orion Nebula with a mass of $8 \times 10^{4} \mathrm{M}_{\odot}$. Extensive maps of ${ }^{13} \mathrm{CO}$ emission (Bally et al. 1987; Bally this vol.; Fukui this vol.) reveal complex filamentary structures throughout the L1641 and L1630 clouds. In addition, the L1641 cloud contains the very dense structures and prolific star formation regions near the Orion Nebula, marked by an intense concentration of $\mathrm{OB}$ stars and embedded infrared sources (Genzel and Stutzki 1989).

\subsubsection{L1641}

Recently, studies of both the dense cores and the young stellar population have been carried out in the $\mathrm{L} 1641$ molecular cloud. A survey of $\mathrm{NH}_{3}$ emission towards IRAS sources in L1641 has identified a number of dense cores within the cloud (Wouterloot, Walmsley, and Henkel 1988). Maps of $\mathrm{NH}_{3}$ reveal that cores in L1641 have significantly larger sizes and linewidths than those in Taurus (Harju, Walmsley and Wouterloot 1990). In addition the L1641 cores have higher kinetic temperatures and larger masses. IRAS-based studies of the young stellar population (Strom et al. 1989) have identified 93 IRAS point sources to be associated with the L1641 cloud. These sources are distributed throughout the cloud, very reminiscent of the distribution of sources seen in Taurus. One should note however that the IRAS selected sample is only complete to $\sim 6 \mathrm{~L}_{\odot}$, much higher than the completeness limit for comparable studies in Taurus.

Based on the above studies, it appears that star formation in L1641 resembles star formation in Taurus, at least in its distribution. However, we know from optical studies that several clusters are also present in this cloud. One well known cluster is the Trapezium cluster. Recently, McCaughrean et al. (1990) have found a total of 480 near-infrared sources in this area, indicating that many of the stars are buried in the dense molecular cloud immediately behind the Trapezium cluster. In addition to this cluster, Strom, Margulis, and Strom (1989b) have found a cluster of $\sim 20$ sources associated with IRAS 05338-0624. It seems that star formation in L1641 also occurs in clusters, similar to star formation in the $\rho$ Ophiuchi cloud. In fact, if we compare the number of embedded sources found in clusters to the total number of embedded sources known to be associated with the L1641 cloud, roughly $85 \%$ would be located in clusters. This number may be an overestimate, 
since the IRAS survey is not sensitive to the entire stellar mass spectrum; more low-mass stars may be distributed throughout the cloud.

\subsubsection{L1630}

Recently, unbiased, systematic and sensitive surveys for dense gas and embedded infrared sources have been obtained in the L1630 molecular cloud. These surveys provide the first complete census of dense cores and embedded young stellar objects (YSOs) within a single molecular cloud and allow us to investigate systematically the relationship between dense cores and their associated young stellar objects.

To identify the dense cores, the L1630 molecular cloud has been surveyed for dense gas in the $\mathrm{J}=2 \rightarrow 1$ transition of CS (Lada, Bally, and Stark 1991). Emission was detected, at a $3 \sigma$ level over $10 \%$ of the area surveyed, revealing a very clumpy structure in the dense gas on $1^{\prime}-2^{\prime}$ scales. Forty-two individual cores were identified at a $5 \sigma$ level above the noise, including all previously known regions of star formation (i.e., NGC 2071, 2068, 2024, 2023, and core number 23, which includes the M78 (HH 19-27) star formation region). Approximately $50 \%$ of the total mass of dense gas is contained within the 5 most massive cores, each with a mass greater than $200 \mathrm{M}_{\odot}$. These 5 massive cores cover a total area of $\sim 2 \mathrm{pc}^{2}$, only $1 \%$ of the total area surveyed, indicating that the dense gas is highly localized.

A near-infrared $(2.2 \mu \mathrm{m})$ survey of a significant portion of the cloud (Lada et al. 1991), including both areas containing CS emission and areas without CS emission, revealed 912 sources having $\mathrm{m}_{K}<13$. At the distance to L1630, this limit corresponds to a $0.6 \mathrm{M}_{\odot}$ main sequence dwarf. Using statistical arguments, Lada et al. (1991) estimated that $\sim 50 \%$ of the sources with $\mathrm{m}_{K}<13$ are background stars. Even with this large contribution from a uniform background distribution, the observed sources appear grouped or clustered. Four embedded clusters were identified, where an embedded cluster is defined as a region in the sky where the source density significantly increases over the background star density. These clusters are associated with the well known star formation regions, NGC 2071, 2068, 2024 , and 2023. The embedded clusters exhibit a range in size $(\mathrm{r}=0.3 \mathrm{pc}$ to 0.9 pc) and number of sources $(\sim 20-300)$. The most spectacular cluster is associated with the HII region NGC 2024. Whereas optical images are dominated by a dark, obscuring dust lane, many sources are present in the $2 \mu \mathrm{m}$ image, along with substantial nebulosity. Lada et al. (1991) count 309 sources having $\mathrm{m}_{K}<14$ located within the cluster boundary.

Surprisingly, Lada et al. (1991) find that the majority of the embedded sources associated with L1630 are located in the four embedded clusters. These clusters contain at least $58 \%$ (if no correction for background stars is made) and possibly as much as $96 \%$ (if correction for background stars is made) of the total number of sources in the molecular cloud! Furthermore, the total area covered by the four embedded clusters is only $18 \%$ of the total region surveyed, indicating that star formation in L1630 is a highly localized process even for stars whose masses are as low as the mass of the sun. 
Comparing the distribution of dense gas and the distribution of embedded infrared sources (see Figure 3), Lada (1990) finds that the embedded clusters are coincident or nearly coincident with 4 of the most massive $\left(M>200 M_{\odot}\right)$ CS cores. Star formation, in this region, is occurring almost exclusively in the most massive cores of dense gas. There is no evidence for any significant star forming activity outside these localized centers. Moreover, $97 \%$ of the sources found in the embedded clusters are associated with only 3 massive CS cores.

The CS and $2.2 \mu \mathrm{m}$ surveys have revealed that the formation of both high and low mass stars in L1630 is occurring mainly in clusters. These clusters in turn are produced by the largest and most massive dense cores in the cloud. These results suggest that in order to understand how most stars in this cloud form, one must understand how massive cores form and produce clusters of stars with relatively high efficiency.

\section{Star Formation Efficiency and the Initial Mass Function}

As described above, the studies of the Taurus, Ophiuchus and Orion complexes have revealed that the distribution of star formation is different in the three clouds. Taurus forms stars individually or in small groups dispersed through the cloud. Ophiuchus and L1641 (Orion A) are forming most of their stars in a single dense cluster, with other regions of more isolated star formation scattered through the cloud. Finally, L1630 (Orion B) is forming most of its stars in three rich clusters, which are spread through the cloud. It is now of interest to see if the differences between the distributions of star formation in these clouds are connected with other differences, such as the star formation efficiency and the initial mass function. Observational determination of both of these is very difficult, but we can make some preliminary comparisons.

In principle, it is straightforward to determine the global star formation efficiency of a molecular cloud complex. This can be done by comparing the total mass of stars with the total mass of stars plus gas $\left[S F E=M_{*}^{\text {tot }} /\left(M_{*}^{\text {tot }}+M_{\text {gas }}\right)\right]$. Of course, the final star formation efficiency of a complex can only be determined when the cloud has finished forming stars. However we can compare the star formation efficiency so far by estimating the mass of already formed stars and the mass of the cloud. For example, in Taurus-Auriga, Kenyon et al. (1990) conclude that there are 100 140 pre-main-sequence stars with $\mathrm{L} \geq 0.5 \mathrm{~L}_{\odot}$; if the median mass is $0.6 \mathrm{M}_{\odot}$ (Larson 1986), then the total mass of stars $\left(M_{*}^{\text {tot }}\right)$ is about $70 \mathrm{M}_{\odot}$. The total mass of the whole complex is $1 \times 10^{4} \mathrm{M}_{\odot}$, yielding a $S F E$ of about $0.7 \%$. In Ophiuchus, Wilking, Lada, and Young (1989) have done a very careful accounting of the stars in the $\rho$ Ophiuchi cluster, concluding that $M_{*}^{\text {tot }}=82 \mathrm{M}_{\odot}$. Since the total mass of the Ophiuchus cloud is also $1 \times 10^{4} \mathrm{M}_{\odot}$, the overall efficiency is about $0.8 \%$ as long as there is not a large contribution from the rest of the complex. Similarly, we can estimate the star forming efficiency in L1641. Based on the infrared surveys of this cloud (Strom et al. 1989; Strom, Margulis and Strom 1989b; McCaughrean et al. 


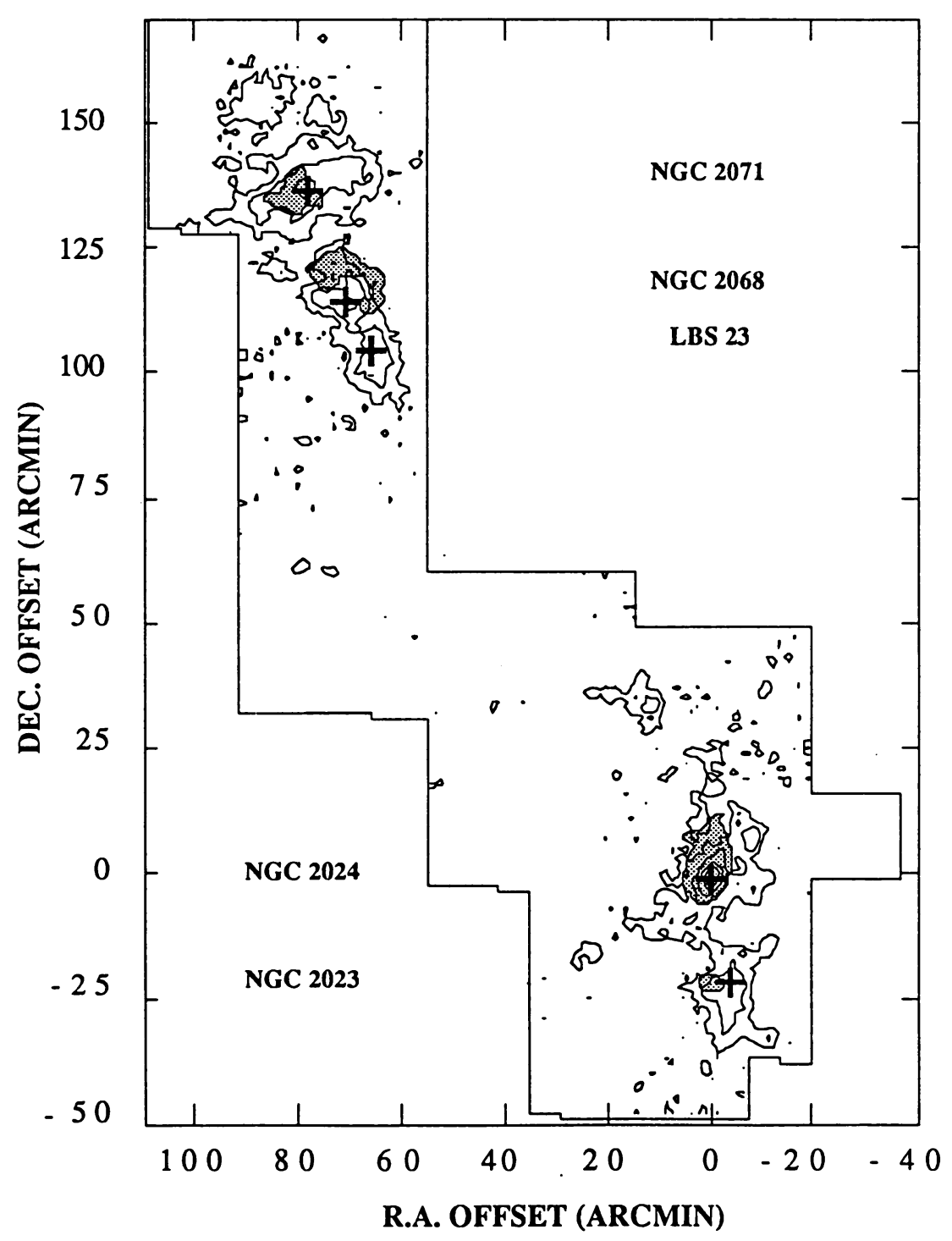

Fig. 3. Locations of the embedded infrared clusters and dense CS cores in the L1630 Molecular Cloud (from Lada 1990). The locations and extents of the embedded stellar clusters are shown as shaded regions and the distribution of dense gas is presented as intensity contours of $C S(2 \rightarrow 1)$ emission. The lowest $C S$ contour is equal to a $9 \sigma$ detection level above the noise $\left(0.8 \mathrm{~K} \mathrm{~km} \mathrm{~s}^{-1}\right.$. In addition, the peak intensity positions of the 5 most massive $C S$ cores $\left(M>200 M_{\odot}\right)$ are represented by crosses. 
1990 ), we estimate that $\sim 600$ young stellar objects are associated with the cloud. For a mean stellar mass of $1 \mathrm{M}_{\odot}$, and $\mathrm{M}_{\text {gas }}$ of $1 \times 10^{5} \mathrm{M}_{\odot}$, we again get an overall $S F E$ of about $0.6 \%$. Finally, estimates of the global star formation efficiency in the L1630 cloud (Lada 1990) are also low, with $S F E \sim 3 \%-4 \%$.

Comparing the estimates for the global star formation efficiency and allowing for the very large uncertainties in all of them, we see that the star formation efficiencies of the three clouds are very similar, with $~ 1 \%$ of the gas in each complex forming stars so far. While these estimates are lower limits to the final $S F E$ of these clouds, they are similar to the estimates for the final $S F E$ of clouds which form open clusters (Leisawitz, Bash, and Thaddeus 1989) and of the cloud which formed the $\lambda$ Orionis association (Duerr, Imhoff, and Lada 1982). These results indicate that only a small fraction of the gas of a typical molecular cloud ever has the conditions required for star formation.

The similarity of the global efficiencies of these three cloud complexes is somewhat surprising in view of the different distributions of star formation discussed earlier. If the only requirement for star formation is that the gas density rise above about $10^{4} \mathrm{~cm}^{-3}$, then the similarity in $S F E$ would suggest that the fraction of the gas with $\mathrm{n} \geq 10^{4} \mathrm{~cm}^{-3}$ is roughly the same for these three complexes. In this case, the difference between them would be that, in Ophiuchus and Orion, the dense gas is collected into much more massive cores than it is in Taurus, resulting in the formation of large clusters rather than small groups. However, the L1630 cloud suggests that the situation is more complex. Five massive $\left(M>200 M_{\odot}\right)$ dense cores exist in the cloud, yet only 3 cores seem to be producing most of the young stellar objects. This is reflected in estimates of the star formation efficiency of the cores. For example, the NGC 2024, NGC 2071 and NGC 2068 cores have $S F E$ ranging from $20 \%-40 \%$, while the NGC 2023 core and core number 23 have $S F E \sim 7 \%$ (Lada 1990). Since we do not yet know the final efficiencies, these differences may only be temporary. If they reflect the final efficiencies, then factors other than having a density $\geq 10^{4} \mathrm{~cm}^{-3}$ may be important. Detailed comparisons of the physical conditions in these different cores are needed to explore this question. It will also be important to assess the local efficiency for the $\mathrm{NH}_{3}$ cores in Taurus, using the same criteria as in L1630 for determining the mass of gas with $\mathrm{n} \geq 10^{4} \mathrm{~cm}^{-3}$.

We can also ask whether the initial mass functions differ among these three regions. The initial mass function of field stars and fully-formed open clusters appears to be characterized by a power law slope in the $d N / d \log \left(M_{*}\right)$ versus $M_{*}$ relation for large masses and a turnover below about $0.5 \mathrm{M}_{\odot}$ (e.g., Scalo 1986, Larson, this vol.). The slope of the power law appears to be about the same $(1.7 \pm 0.5)$ in most clusters and in the field. Is this result consistent with the anecdotal statements from the star formation field that "Orion forms high mass stars" while "Taurus forms low mass stars"? The optical studies by Cohen and Kuhi (1979) found values for the slopes of $1.5(1.2-2.5)$ in Taurus and $1.35(1.3-1.5)$ in Orion, both consistent with the above range, as well as with each other. Is the difference then only that Orion is forming more stars overall, so the high end of the IMF is better 
sampled than in Taurus? This question is hard to answer at this point, but the lack of massive stars in Taurus is consistent with the total number of stars and a standard IMF.

The recent surveys at $2 \mu \mathrm{m}$ have the potential for clarifying these issues, since they should be much more complete and less biased than the optical surveys. Such surveys can be used to construct cumulative luminosity functions for the clusters, in which the logarithm of $N$, the number of sources brighter than a given $2 \mu \mathrm{m}$ magnitude, $m_{K}$, is plotted versus $m_{K}$. The four clusters in the L1630 cloud have luminosity functions which are well described by power laws for $m_{K}$ above the completeness limit. The slopes in the $\log (N)-m_{K}$ relation are consistent for the three rich clusters, with a value of $0.38 \pm 0.03$. We can compare this result with what would be predicted from a given IMF with the following assumptions : the $2 \mu \mathrm{m}$ emission arises only from stellar photospheres, the extinction at $\mathrm{K}$ is either negligible or at least does not depend on $\mathrm{m}_{K}$, and the stars are on the main sequence. The result which would be predicted from a slope in the initial mass function of $1.7 \pm 0.5$ would be $0.34 \pm 0.08$, consistent with the data.

The near-infrared surveys can also be used to look for a turnover in the IMF at low masses, which would produce a flattening in the cumulative luminosity function, but considerable caution is needed. If the lowest mass stars are not yet on the main sequence, their luminosity will exceed the luminosity they will have when they reach the main sequence. Thus, a flattening in the cumulative luminosity function, interpreted in terms of a main - sequence mass-luminosity relation, would overestimate the mass at which the IMF turns over. There is some indication of a flattening in the luminosity function in the L1630 clusters, but it occurs around $m_{K}$ of 12 , near the completeness limit of 13 , making it rather uncertain. DePoy et al. (1990) have obtained more sensitive $\left(m_{K} \sim 15\right)$ data on one of the clusters (NGC2023) and confirm the flattening. Similarly, McCaughrean et al. (1990) found a possible deficit of stars fainter than $m_{K}=11.5$ in the Trapezium cluster. They interpreted their data in terms of pre-main-sequence stars with ages of $10^{6}$ years; in this interpretation, the mass function peaks strongly at $0.3 \mathrm{M}_{\odot}$, with little or no evidence for stars less massive than $0.1 \mathrm{M}_{\odot}$. While these conclusions may be sensitive to the age (and to the assumption of a single age for all the sources), they do suggest that the lower mass limit in the Trapezium cluster is not very different from that in Taurus, and that the initial mass function of this forming cluster is similar to that of more evolved clusters.

How does Ophiuchus fit into this question? Elias (1978a) commented that, while the most massive star in both Ophiuchus and Taurus is a B2 star, Ophiuchus boasts about 20 stars of spectral type A0 or earlier, compared to only 2 or 3 in Taurus. Wilking, Lada, and Young (1989) have constructed a luminosity function for the $\rho$ Ophiuchi Cloud core and conclude that the luminosity function deviates from that expected from the IMF of field stars by 2.5 to $3.5 \sigma$ in the sense of a deficit of intermediate luminosity stars. They also find a deficit at low luminosities $\left(\mathrm{L}<1 \mathrm{~L}_{\odot}\right)$, but attribute this to incompleteness. Recently, Kenyon et al. (1990) 
compared the luminosity functions of Taurus and the $\rho$ Ophiuchi core. They found that Ophiuchus contains many more deeply embedded (Class I) sources with $\mathrm{L}>$ $10 \mathrm{~L}_{\odot}$ than does Taurus, while the T-Tauri stars (class II sources) in each cloud have similar luminosity functions.

The low end of the luminosity function in Ophiuchus has been somewhat more controversial recently. Based on a survey of a small region in the dense core of the cloud, Rieke, Ashok, and Boyle (1989) found a turnover at $\mathrm{m}_{K}$ of 9 ; considering the smaller distance to this cloud, this limit would correspond to a turnover in absolute magnitude at a value similar to that in Orion. However, Barsony et al. (1989), having surveyed a larger area, found no evidence of a turnover to $\mathrm{m}_{K}$ of 14. The discrepancy between the two surveys seems to be caused by an absence of faint sources in the region surveyed by Rieke, Ashok, and Boyle; whether this is simply due to chance or to spatial variations in the luminosity function is not yet clear. One possibility is magnitude segregation, in which the fainter sources are more widely distributed than the brighter sources; there is some evidence for this in the L1630 clusters (Lada et al. 1991); and it may correspond to mass segregation seen in some more evolved clusters (e.g., Scalo 1986).

If the extinction at $\mathrm{K}$ is negligible, the limit of Barsony et al. (1989) would correspond to an absolute $\mathrm{K}$ magnitude of about 8 , implying very low mass stars, even in the unlikely event that they are on the main sequence. These sources may fill in the low luminosity part of the luminosity function in Ophiuchus, making it look more like the function in Taurus (see fig. 8 in Kenyon et al. 1990).

At this point, the evidence for variations in the IMF among these three regions is weak; many of the apparent differences can be traced to the larger cloud masses in the Orion cloud, which allow more stars to form, more fully sampling the IMF (see also Elmegreen 1983). Before suggested differences can be taken seriously, much more work needs to be done to correct for background sources, to rule out possible dust emission at $2 \mu \mathrm{m}$, and to pin down extinction corrections. In addition, more complete and sensitive surveys would help to remove some selection effects. In some dense regions, the extinction is clearly large, even at $2 \mu \mathrm{m}$, and observations at longer wavelengths are needed to determine the number of sources missed in the $2 \mu \mathrm{m}$ surveys. The IRAS survey can help with this problem, but its low resolution implies that extensive follow-up studies are necessary. While evolutionary effects make it difficult to compare postulated IMF's to observed luminosity functions, the data so far seem to be consistent with all three regions having IMF's similar to those of evolved clusters and field stars.

\section{Studies of Individual Sources}

Having compared the star formation on a global scale in these three nearby clouds, we now turn our attention to studies of individual regions of star formation within each cloud. While many such regions have been studied, we will concentrate on one region in each cloud. Comparison between these regions will give us a preliminary 
look at the mode of star formation in the different clouds, but comparison of more regions in each cloud should eventually provide a more complete picture.

To provide a common ground for comparison of these individual regions in each of the three clouds, we will compare the observational results to the predictions of theoretical models of star formation, as developed recently by Shu and his collaborators (Shu, Adams, and Lizano 1987). In this picture, low mass star formation occurs in relatively isolated cores in molecular clouds. These cores contract slowly to form a density structure similar to that of an isothermal sphere; that is, $n(r) \propto r^{-2}$ (Shu 1977). At some point, collapse may begin in the center of the core; as a wave of infall propagates outward, an inside-out collapse occurs. If the infall wave propagates at speed $a$ (in the simplest case, this is the isothermal sound speed), its radius is simply related to the time since collapse began by $r_{2}=a t$. Inside $r_{2}$, the density distribution will be modified to approximate $n(r) \propto r^{-1.5}$. These predictions are similar to those of earlier dynamical collapse calculations (Larson 1969; Penston 1969), but the predicted velocity field is significantly different. Observations of $\mathrm{H}_{2} \mathrm{CO}$ in B335, a small star-forming globule, are in excellent agreement with the predictions of the Shu picture, while clearly in disagreement with those of Larson-Penston type collapse (Zhou et al. 1990). If rotation is initially present, it further modifies the collapse in the innermost regions; a disk is likely to form inside the centrifugal radius, $R_{c}$ (Terebey, Shu, and Cassen 1984).

With this background in mind, we will compare three star formation regions : L1551 IRS 5 (in the Taurus complex); IRAS 16293-2422 (in the Ophiuchus cloud); and NGC2071 (in the Orion cloud, L1630). The first two regions are forming low mass stars, based on their luminosities of $30 \mathrm{~L}_{\odot}$ and $27 \mathrm{~L}_{\odot}$, respectively, while NGC2071 has a luminosity of $520 \mathrm{~L}_{\odot}$, suggesting a star of considerably higher mass.

\section{1. L1551 IRS5 (TAURUS COMPLEX)}

L1551 IRS5 is the prototypical bipolar outflow source (Snell, Loren, and Plambeck 1980) and the L1551 cloud (a component of the Taurus complex) appears to have a density distribution of $n(r) \propto r^{-2}$ on scales of a few tenths of pc (Snell 1981), consistent with the the initial configuration having been an isothermal sphere. The source has been modeled in detail by Adams, Lada, and Shu (1987), who predicted that infalling matter extends from 40 to $3500 \mathrm{AU}$, corresponding to angles of $0.3^{\prime \prime}$ to $21^{\prime \prime}$ at the distance of $160 \mathrm{pc}$ to this particular cloud (Snell 1981). Inside $40 \mathrm{AU}$, they predicted that a disk should exist, extending inward to the stellar surface and argued that the emission from this disk compensated for a deficit of emission at mid-infrared wavelengths $(5-20 \mu \mathrm{m})$ that appeared in spherically symmetric models (Adams and Shu 1985, 1986). The star itself has been studied by observing photospheric features at $2 \mu \mathrm{m}$ (Carr, Harvey, and Lester 1987) and at shorter wavelengths via scattered light (Mundt et al. 1985). The spectra show the characteristic features of an FU Orionis object. These objects can be understood as pre-main-sequence stars surrounded by massive accretion disks, with the outbursts 
explained by disk instabilities (Hartmann and Kenyon 1985). Thus, there is some indirect evidence for a disk, as envisioned by Adams, Lada, and Shu (1987). The direct evidence is somewhat more equivocal : both the pattern of scattered light at $2 \mu \mathrm{m}$ (e.g., Campbell et al. 1988) and the high-resolution $\mathrm{C}^{18} \mathrm{O}$ map (Sargent et al. 1988) indicate elongated structures, but on scales considerably larger than the $40 \mathrm{AU}$ size predicted by Adams, Lada, and Shu (1987).

The region predicted to contain infalling material has been studied recently with high-resolution (10" to 20") far-infrared observations (Butner et al. 1991a); the source is clearly resolved at $100 \mu \mathrm{m}$ (with a beam-deconvolved size of about $\left.11^{\prime \prime}\right)$, but not at $50 \mu \mathrm{m}$. Detailed comparison of the far-infrared scans with predictions of the the intensity distribution from the Adams, Lada, and Shu (1987) model (Adams, private communication) show good agreement. The data also agree well with the predictions of radiative transport models with spherically symmetric density distributions characteristic of infall $\left(n(r) \propto r^{-\alpha}\right.$ with $\left.\alpha=1.5\right)$; thus the departures from spherical symmetry, including the disk, in the detailed models of Adams, Lada, and Shu (1987) do not affect the far-infrared emission at the scales probed by the data. Similar models with other density laws $(\alpha=1.0$ or 2.0$)$ clearly do not fit the data well. Butner et al. (1991a) concluded that their data were in reasonable agreement with the model of Adams, Lada, and Shu (1987), specifically with the prediction of infalling material on scales of a few thousand AU. Confirmation of this result with spectroscopic data is clearly needed to establish firmly the presence of infall, but confusion with the outflowing gas makes this a tricky project.

The Adams, Lada, and Shu (1987) interpretation of the mid-infrared spectrum as evidence for a disk in L1551 is more problematic. In order to produce the observed emission at far-infrared wavelengths, the dust opacity must be very high at mid-infrared wavelengths, assuming the dust opacities used by Adams, Lada, and Shu (1987), which are similar to those of Draine and Lee (1984). The mid-infrared radiation from a disk will be completely attenuated and re-emitted at longer wavelengths. Spherically symmetric models of this source produce very deep silicate features and a pronounced mid-infrared deficit, whether or not disks are included in the spectrum of the internal energy source (Butner, Natta, and Evans 1991), as long as the dust opacities are assumed to be like those of Draine and Lee (1984). There are two possible answers to this problem : one is that the mid-infrared radiation is scattering out the poles of the outflow, in which case it should be polarized, like the near-infrared emission (e.g., Hodapp et al. 1988); the second is that the actual dust opacities have a lower ratio of mid-infrared opacity to far-infrared opacity than do those of Draine and Lee (1984). Dust opacities with this property were advocated by Mathis, Mezger, and Panagia (1983), and Butner et al. (1991a) found that they provide better agreement with the spectral energy distribution of L1551 IRS 5 than do those of Draine and Lee (1984) or of Adams, Lada, and Shu (1987).

Leaving aside scattered light, with its own interpretational ambiguities (for example, compare the conclusions of Beckwith et al. (1984) with those of Beckwith 
et al. (1989) or those of Leinert and Haas (1987) with those of Koresko, Beckwith, and Sargent (1989)), the only way disks in deeply embedded sources, like L1551 IRS5, are likely to be seen directly is at very long wavelengths, where both the attenuation and the emission from dust in the infalling envelope are minimized. In fact, Keene and Masson (1990) have found a compact continuum source in millimeter-wavelength interferometer observations. This compact source cannot be reproduced in the infalling envelope models of Butner et al. (1991a), clearly indicating the need for a separate component. While Keene and Masson (1990) did not resolve the source, they interpreted it as a disk with an outer radius of $45 \mathrm{AU}$ $\left(0.3^{\prime \prime}\right)$, very similar to the predicted $R_{c}$ in the Adams, Lada, and Shu (1987) model. The surrounding envelope does produce emission at these wavelengths, but this envelope emission is very extended. The most convincing observational signature of compact structures, plausibly interpreted on theoretical grounds as disks, is then a ratio between the emission in large and small beams at long (e.g., millimeter) wavelengths which requires the existence of a substantial compact component.

\section{2. IRAS16293-2422 ( L1689 - OPHIUCHUS)}

This remarkable IRAS source lies in the L1689 cloud, east of the main body of the Ophiuchus cloud, where most of the near-infrared surveys discussed above were performed, but still part of the Ophiuchus complex (Loren 1989a). Since the source has a luminosity of $27 \mathrm{~L}_{\odot}$ (Mundy, Wilking, and Myers 1986), very similar to that of L1551 IRS 5, it provides an interesting comparison. The source was found to have wings and self-absorption in the CS $\mathrm{J}=5 \rightarrow 4$ line, with a pattern suggestive of infall (Walker et al. 1986). Later studies suggested that the self-absorption was caused by an extended, foreground absorbing layer, calling into question the kinematic evidence for infall (Menten et al. 1987); instead, rotation appears to be present (Menten et al. 1987; Mundy, Wootten, and Wilking 1990). The source contains at least one outflow (Wootten and Loren 1987; Walker et al. 1988), with an axis roughly perpendicular to the axis of elongation of the continuum emission at $3 \mathrm{~mm}$ (Mundy, Wilking, and Myers 1986). The strong emission at millimeter wavelengths indicates that there is a very opaque core surrounding the source, making it invisible at wavelengths less than about $20 \mu \mathrm{m}$ (Mundy, Wilking, and Myers 1986). Radio continuum measurements at $6 \mathrm{~cm}$ and $2 \mathrm{~cm}$ revealed two sources, located near peaks in the $3 \mathrm{~mm}$ continuum distribution and separated by about $5^{\prime \prime}$ (750 AU), leading Wootten (1989) to suggest that the system was a protobinary star.

Using the same far-infrared scanning system as was used for L1551, Butner et al. (1991b) have resolved the source at both 50 and $100 \mu \mathrm{m}$. Unlike L1551 IRS 5, the far-infrared data are not consistent with a density distribution characteristic of infall, but instead suggest relatively flat density laws $(\alpha=0$ to 0.5$)$. This very different behavior, compared to that found in L1551 IRS5, may be related to the possible presence of a protobinary system (Wootten 1989; Mundy, Wootten, and Wilking 1990). Clearly, this source has much more circumstellar material, and large-scale rotation appears to play a more important role, compared to the case 
of L1551 IRS5. Certainly the Adams, Lada, and Shu (1987) picture does not apply well to this source, but this discrepancy may be more related to the rather unusual properties of this source than to differences between the Taurus and Ophiuchus regions.

\section{3. NGC2071 (L1630 - ORION)}

Even though the models of Adams, Lada, and Shu (1987) were developed for low mass stars, it is interesting to test the basic ideas for stars of somewhat higher mass and in different environments. In particular, we may expect star formation to proceed somewhat differently in a clustered, rather than non-clustered, environment. The infrared source in NGC2071 offers such an opportunity. In a part of the L1630 cloud which is at a distance of 390 pc (Anthony-Twarog 1982), it lies in one of the clusters of $2 \mu \mathrm{m}$ sources discussed above (Lada et al. 1991); the region we are discussing lies north of the NGC2071 reflection nebula and contains a small group of $10 \mu \mathrm{m}$ sources (Persson et al. 1981), of which IRS 1 is clearly dominant at $10 \mu \mathrm{m}$. If all the far-infrared emission is attributed to IRS 1 , the luminosity of this source is $520 \mathrm{~L}_{\odot}$, suggesting a star of about $5 \mathrm{M}_{\odot}$ (Butner et al. 1990).

Far-infrared scans of this source resolved it at both 50 and $100 \mu \mathrm{m}$ (Butner et al. 1990). Like L1551 IRS5, the far-infrared scans are consistent with a power law density distribution, decreasing with distance from the energy source. This source was not specifically modeled by Adams, Lada, and Shu (1987), but the basic ideas can still be tested. To make a more specific comparison, values for the centrifugal radius $\left(R_{c}\right)$ and the radius of the infall region $\left(r_{2}\right)$ can be estimated (see Evans 1991). Assuming a stellar mass of $5 \mathrm{M}_{\odot}$ (Zhou, Evans, and Mundy 1990), the same value for the isothermal sound speed as in the Adams, Lada, and Shu (1987) models of L1551 IRS5, and a rotation rate of $\Omega=5.8 \times 10^{-14} \mathrm{~s}^{-1}$ (Takano et al. 1986), one finds $R_{c}=1900 \mathrm{AU}$ and $r_{2}=1.5 \times 10^{4} \mathrm{AU}$, which correspond to $5^{\prime \prime}$ and $38^{\prime \prime}$, respectively, at the distance of NGC2071. Thus, the far-infrared scans should once again be probing primarily the infall region. In fact, comparison of the data to radiative transport calculations with various choices for $r_{2}$ and $\alpha$ indicates that the best fit for the inner radius of the spherical distribution is $5^{\prime \prime}$. The value of $\alpha$ depends somewhat on the choice of dust opacities : for the Draine and Lee (1984) opacities, the best-fitting $\alpha$ is 2.0, while the Mathis, Mezger, and Panagia (1983) opacities favor $\alpha$ of 1.5. The Mathis, Mezger, and Panagia (1983) dust opacities provide a somewhat better fit to the spectral energy distribution, suggesting that $\alpha=1.5$ is the more likely case, thus making this source appear to be consistent with the ideas of Adams, Lada, and Shu (1987). However, the normalizing density can also be predicted from the sound speed and the mass of the star; the result is $n_{i}=8 \times 10^{4} \mathrm{~cm}^{-3}\left(n_{i} \equiv n\left(R_{c}\right)\right)$, whereas the far-infrared data suggest considerably higher densities $\left(n_{i}=7 \times 10^{5} \mathrm{~cm}^{-3}\right.$ for the Mathis, Mezger, and Panagia (1983) opacities, and $8 \times 10^{6} \mathrm{~cm}^{-3}$ for the Draine and Lee (1984) opacities). At least some modifications to the Adams, Lada, and Shu (1987) picture appear to be necessary for this source. These modifications may be generally necessary for more massive 
stars or stars which are forming in clusters.

Since the far-infrared emission is proportional to the column density of dust, the derivation of $n_{i}$ is rather indirect; it depends on the assumption of spherical symmetry, the assumed dust opacities, and the absence of clumpy structure. It is possible to probe the volume density directly, using molecular line observations. A study of 4 transitions of CS with resolution similar to that of the far-infrared data has been obtained (Zhou et al. 1991). All transitions peak strongly on the infrared position, and the maps are all quite round, indicating that spherical symmetry is a reasonable approximation. The data were compared with the predictions of non-LTE excitation and radiative transport models, using the microturbulent approximation, which assumed various density gradients. The temperature distribution was taken from the radiative transport models of the dust emission (Butner et al. 1990), since the densities are high enough to equilibrate the gas kinetic temperature to the dust temperature. The inner radius of the models was set to $5^{\prime \prime}$, based on the results of the dust modeling, leaving the normalizing density $\left(n_{i}\right)$, the power law in the density gradient $(\alpha)$, and the CS abundance as free parameters. The best-fitting models had $n_{i}=3 \times 10^{6} \mathrm{~cm}^{-3}, \alpha=1.3$, and a CS abundance of $2 \times 10^{-9}$. This model also predicted the correct strength of the $\mathrm{C}^{34} \mathrm{~S} \mathrm{~J}=7 \rightarrow 6$ emission. These values are reasonably consistent with those found from the far-infrared analysis, considering the various uncertainties, although models with the density distributions found by the far-infrared analyses clearly gave worse fits to the CS data.

The CS data favors a density four times larger than that found by the far-infrared modeling with the dust opacities of Mathis, Mezger, and Panagia (1983); this difference may be caused by uncertainties in either the collision rates for CS excitation or the dust opacities. In particular, a smaller dust opacity in the far-infrared would require a larger column density to match the observed far-infrared emission. The other possible explanation is that the infalling cloud is not completely smooth, but contains some clumpy structure; since the molecular emission is sensitive to the volume density, as well as the column density, it will be enhanced relative to the dust emission in the clumps, leading to a higher density derived from the molecules than from the dust.

Lest it be thought that all molecular species trace the distribution of material equally well, it should be noted that a high resolution study of $\mathrm{NH}_{3}$ shows a distribution very different from that of the dust or the CS (Kawabe et al. 1989; Zhou, Evans, and Mundy 1990). The $\mathrm{NH}_{3}$ has a very elongated distribution. Based on the temperature and velocity structure in the $\mathrm{NH}_{3}$, Zhou, Evans, and Mundy (1990) conclude that most of the $\mathrm{NH}_{3}$ is distributed in a ring of radius $0.1 \mathrm{pc}$, outside the region of $\mathrm{CS}$ and dust emission. Only a small amount of $\mathrm{NH}_{3}$ emission arises from gas close (about $0.01 \mathrm{pc}$ ) to the star; unlike the bulk of the $\mathrm{NH}_{3}$, this gas has a velocity structure consistent with Keplerian rotation about a $5 \mathrm{M}_{\odot}$ star. The abundance of $\mathrm{NH}_{3}$ in the region of dust and CS emission must be about 100 times lower than in the outer parts or in typical low density regions, suggesting that it 
is frozen onto dust grains. A similar result was found in IRAS16293-2422 (Mundy, Wootten, and Wilking 1990). Thus, $\mathrm{NH}_{3}$ is a poor tracer of the densest gas in these two sources and should be regarded with caution in other regions as well (see also Zhou et al. 1989).

\section{Conclusions}

The large-scale infrared surveys of the three clouds described above have confirmed many, but not all, of the ideas derived from earlier studies. The distribution of star formation does appear to be different in the three clouds: Taurus forms stars individually or in small groups dispersed through the cloud; Ophiuchus and L1641 (Orion A) are forming most of their stars in a single dense cluster, with other regions of more isolated star formation scattered through the cloud. However, L1630 (Orion B) is following yet another pattern, with most star formation occurring in 3-4 clusters, which are spread through the cloud.

Lada (1990) and Lada, Strom and Myers (1991) have suggested that Taurus and L1630 represent two distinct modes of star formation : isolated and clustered star formation. It is also possible that they represent extremes in a continuum of star formation styles, ranging from individualistic (Taurus) to gregarious (L1630). To understand the origin of these different modes or styles, we will need more complete studies of the physical conditions in these three clouds. For example, complete CS maps of Taurus-Auriga and Ophiuchus, comparable to those obtained for L1630 (Lada, Bally, and Stark 1991) would reveal whether the differences in star formation patterns can be traced to differences in the distribution and nature of the molecular gas. Even without these maps, there is evidence in all three regions that star formation requires fairly dense gas $\left(\mathrm{n}>10^{4} \mathrm{~cm}^{-3}\right)$; a substantial mass of such dense gas $\left(M>200 M_{\odot}\right)$ seems necessary for forming a cluster, but perhaps not sufficient, since two massive cores in L1630 are not yet forming substantial clusters.

Despite the differences in the distribution of star formation in these three complexes, the net result seems surprisingly similar. Each complex seems to have turned about $1 \%$ of its total mass into stars so far, resulting in a global efficiency which is very similar to those deduced from studies of fully formed open clusters and associations. Furthermore, the initial mass functions of open clusters and field stars, when used to predict the luminosity functions of embedded infrared sources, produce reasonable consistent results, within the substantial uncertainties. It may be that the vastly different stellar content of Orion (many O and B stars) and Taurus (mostly low mass stars) can be explained simply by the larger mass (and thus greater chance of forming massive stars) of the Orion complex.

Detailed studies of individual star formation regions in the three clouds reveal some interesting differences. While the density distribution around L1551 IRS 5 (in Taurus) agrees very well with theoretical models, the opacity is clearly higher in the nominally similar source, IRAS16293-2422 (in Ophiuchus) than would be expected 
from theoretical models or from comparison to Taurus. In the Orion complex, the NGC2071 region clearly has a higher density than does the gas around L1551 IRS 5 ; it is also higher than predicted from theory. This may be related to the fact that the forming star is more massive and that it is forming in the environment of one of the L1630 clusters. Similar studies of other regions in these three clouds are needed to distinguish which of these differences arise from differences between the clouds and which are peculiar to these three sources.

\section{Acknowledgements}

We thank the members of the Organizing Committee for their invitation to present this paper. We also thank Charles Lada, Lee Mundy and Harold Butner for useful comments and discussions. Portions of the research described here were supported in part by NSF Grant AST 88-15801, NASA grant NAG 2-420, NASA Training Grant NGT 50320 and by grants from the W. M. Keck Foundation and Texas Advanced Research Program to the University of Texas at Austin.

\section{References}

Adams, F. C., Lada, C. J., and Shu, F. H. 1987, Ap.J., 312, 788.

Adams, F. C., and Shu, F. H. 1985, Ap. J., 296, 655.

Adams, F. C., and Shu, F. H. 1986, Ap. J., 308, 836.

Anthony-Twarog, B. J. 1982, Astron. J., 87, 1213.

Bally, J., Langer, W. D., Wilson and R. W., Stark, A. A. 1987, Ap. J. (Letters), 312, L45.

Barsony, M., Burton, M. G., Russell, A. P. G., Carlstrom, J. E., and Garden, R. 1989, Ap. J. (Letters), 346, L93.

Beckwith, S. V. W., Sargent, A. I., Koresko, C. D., and Weintraub, D. A. 1989, Ap. J., 343, 393.

Beckwith, S., Zuckerman, B., Skrutskie, M. F., and Dyck, H. M. 1984, Ap. J., 287, 793.

Beichman, C. A., Myers. P. C., Emerson, J. P., Harris, S., Mathieu, R. D., Benson, P. J. and Jennings, R. E. 1986, Ap. J., 307, 337.

Benson, P. J. and Myers, P. C. 1989, Ap. J. Suppl., 71, 89.

Bertiau, F. C. 1958, Ap. J., 128, 533.

Butner, H. M., Evans, N. J., II, Harvey, P. M., Mundy, L. G. and Natta, A. 1990, Ap. J., 364, 164.

Butner, H. M., Evans, N. J., II, Lester, D. F., Levreault, R. M. and Strom, S. E. 1991a, Ap.J., in press.

Butner, H. M., Evans, N. J., II, Lester, D. F., Harvey, P. M., Mundy, L. G., and Campbell, M. F. 1991b, Ap.J., in preparation.

Butner, H. M., Natta, A., and Evans, N. J., II 1991, Ap. J., in preparation.

Carr, J. S., Harvey, P. M., and Lester, D. F. 1987, Ap. J. (Letters), 321, L71. 
Campbell, B., Persson, S. E., Strom, S. E., and Grasdalen, G. 1988, Astron. J., 95, 1173.

Cernicharo, J. Bachiller, R., and Duvert, G. 1985, Astr. Ap. Suppl., 149, 273.

Chini, R. 1981, Astr. Ap., 99, 346.

Cohen, M., and Kuhi, L. V. 1979, Ap. J. Suppl., 41, 743.

de Geus, E. J., de Zeeuw, P. T., and Lub, J. 1989, Astr. Ap., 216, 44.

de Geus, E., Bronfman, L., and Thaddeus, P. 1990, Astr. Ap., in press.

DePoy, D., Lada, E. A., Gatley, I., and Probst, R. 1990, Ap. J., in press.

Draine, B. T., and Lee, H. M. 1984, Ap. J., 285, 89.

Dolidze, M. V., and Arakelyan, M. A. 1959, Soviet Astr. - AJ, 3, 434.

Duerr, R., Imhoff, C. L. and Lada, C. J. 1982, Ap. J., 261, 135.

Elias, J. H. 1978a, Ap. J., 224, 857.

Elias, J. H. 1978b, Ap. J., 224, 453.

Elmegreen, B. G. 1983, Mon. Not. Roy. Astr. Soc., 203, 1011.

Evans, N. J., II 1991, in Frontiers of Stellar Evolution, ed. D. L. Lambert (San Francisco: Astronomical Society of the Pacific), in press.

Fuller, G. A. and Myers, P. C. 1987, in Physical Processes in Interstellar Clouds, ed. G. E. Morfill and M. Scholer (Dordrecht : D. Reidel), p.137.

Genzel, R. Reid, M. J., Moran, J. M., and Downes, D. 1981, Ap. J., 244, 884.

Genzel, R., and Stutzki, J. 1989, Ann. Rev. Astr. Ap., 27, 11.

Grasdalen, G. L., Strom, K. M., and Strom, S. E. 1973, Ap. J. (Letters), 184, L53.

Harju, J., Walmsley, C. M. and Wouterloot, J. G. A. 1990, Astron. E Astro., submitted.

Hartmann, L., and Kenyon, S. J. 1985, Ap. J., 299, 462.

Herbig, G. H., and Rao, N. K. 1972, Ap. J., 174, 401.

Hodapp, K., Capps, R. W., Strom, S. E., Salas, L., and Grasdalen, G. 1988, Ap. J., 355, 814.

Haro, G. 1949, A. J., 54, 188.

Ichikawa, T., and Nishida, M. 1989, A. J., 97, 1074.

Kawabe, R., Kitamura, Y., Ishiguro, M., Hasegawa, T., Chikada, Y., and Okamura, S. K. 1989, in Structure and Dynamics of the Interstellar Medium, IAU Colloquium 120, ed. G. Tenorio-Tagle, M. Moles, and J. Melnick (Heidelberg : Springer-Verlag), p.254.

Keene, J., and Masson, C. R. 1990, Ap. J., 355, 635.

Kenyon, S. J., Hartmann, L. W., Strom, K., M. and Strom, S. E. 1990, A. J., 99, 869.

Kleiner, S. C. and Dickman, R. L. 1984, Ap. J., 286, 255.

Kleiner, S. C. and Dickman, R. L. 1985, Ap. J., 295, 466.

Klose, S. 1986, Ap. Space Sci., 128, 135.

Koresko, C. D., Beckwith, S. V. W., and Sargent, A. I. 1989, Astron. J., 98, 1394.

Lada, C. J. and Wilking, B. A. 1984, Ap. J., 287, 610.

Lada, E. A. 1990, Ph.D. thesis, University of Texas at Austin.

Lada, E. A., DePoy, D., Evans, N. J., II, and Gatley, I. 1991, Ap. J., in press. 
Lada, E. A., Bally, J. and Stark, A. A. 1991, Ap. J., in press, Feb. 20.

Lada, E. A., Strom, K. M. and Myers, P. C. 1991, in Protostars and Planets III, ed.

E. H. Levy and J. Lunine (Tucson: University of Arizona Press), in press.

Larson, R. B. 1969, Mon. Not. Roy. Astr. Soc., 145, 271.

Larson, R. B. 1982, Mon. Not. Roy. Astr. Soc., 200, 159.

Larson, R. B. 1986, in Stellar Populations, ed. C. A. Norman, A. Renzini, and M.

Tosi (Cambridge : Cambridge University Press), p.101.

Leinert, Ch., and Haas, M. 1987, Astr. Ap. (Letters), 182, L47.

Leisawitz, D., Bash, F. N., and Thaddeus, P. 1989, Ap. J. Suppl., 70, 731.

Loren, R. B. 1989a, Ap. J., 338, 902.

Loren, R. B. 1989b, Ap. J., 338, 925.

Loren, R. B., Sandqvist, A., and Wootten, H. A. 1983, Ap.J., 270, 620.

Loren, R. B., Wooten, H. A., and Wilking, B. A. 1990, Ap. J, submitted.

Maddalena, R. J., Morris, M., Moscowitz, J., and Thaddeus, P. 1986, Ap. J., 303, 375.

Mathis, J. S., Mezger, P. G., and Panagia, N. 1983, Astr. Ap., 128, 212.

McCaughrean, M., Zinnecker, H., Aspin, C., McLean, I. 1990, in Astrophysics with Infrared Arrays, ed. R. Elston (Tucson: University of Arizona Press), in press.

Menten, K. M., Serabyn, E., Güsten, R., and Wilson, T. L. 1987, Astr. Ap. (Letters), 177, L57.

Mundt, R., Stocke, J., Strom, S. E., Strom, K. M., and Anderson, E. R. 1985, Ap. J. (Letters), 297, L41.

Mundy, L. G., Wilking, B. A., and Myers, S. T. 1986, Ap. J. (Letters), 311, L75.

Mundy, L. G., Wootten, H. A., and Wilking, B. A. 1990, Ap. J., 352, 159.

Myers, P. C. and Benson, P. J. 1983, Ap. J., 266, 309.

Myers, P. C., Heyer, M., Snell, R. L., and Goldsmith, P. F. 1988, Ap. J., 324, 907.

Myers, P. C., Linke, R. A., and Benson, P. J. 1983, Ap. J., 266, 309.

Penston, M. V. 1969, Mon. Not. Roy. Astr. Soc., 144, 425.

Persson, S. E., Geballe, T. R., Simon, T., Lonsdale, C. J. and Baas, F. 1981, Ap. $J$. (Letters), 251, L85.

Rieke, G. H., Ashok, N. H., and Boyle, R. P. 1989, Ap. J. (Letters), 339, L71.

Sargent, A. I., Beckwith, S., Keene, J., and Masson, C. 1988, Ap. J., 333, 936.

Scalo, J. 1986, Fundam. Cosmic Phys., 11, 1.

Scalo, J. 1990, in Physical Processes in Fragmentation and Star Formation, ed. R. Capuzzo-Dolcetta, C. Chiosi, and A. Di Fazio (Netherlands : Kluwer Academic publishers), p.151-177.

Shu, F. H. 1977, Ap.J., 214, 488.

Shu, F. H. 1991, in Frontiers of Stellar Evolution, ed. D. L. Lambert (San Francisco : Astronomical Society of the Pacific), in press.

Shu, F. H., Adams, F. C., and Lizano, S. 1987, Ann. Rev. Astr. Ap., 25, 23.

Snell, R. L. 1981, Ap.J. Suppl., 45, 121.

Snell, R. L., Loren, R. B., and Plambeck, R. L. 1980, Ap. J. (Letters), 239, L17. 
Strom, K. M., Margulis, M., and Strom, S. E. 1989a, Ap. J. (Letters), 345, L79.

Strom, K. M., Margulis, M., and Strom, S. E. 1989b, Ap. J. (Letters), 346, L33.

Strom, K. M., Newton, G., Strom, S. E., Seaman, R. L., Carrasco, L., CruzGonzalez, I., Serrano, A., and Grasdalen, G. L. 1989, Ap. J. Suppl., 71, 183.

Struve, O. and Rudkjobing, M. 1949, Ap. J., 109, 92.

Takano, T., Stutzki, J., Fukui, Y., and Winnewisser, G. 1986, Astr. Ap., 167, 333.

Terebey, S., Shu, F. H., and Cassen, P. 1984, Ap. J., 284, 529.

Ungerechts, H. and Thaddeus, P. 1987, Ap. J. Suppl., 63, 645.

Vrba, F. J., Strom, K. M., Strom, S. E., and Grasdalen, G. L. 1975, Ap. J., 197, 77.

Walker, C. K., Lada, C. J., Young, E. T., Maloney, P. R., and Wilking, B. A. 1986, Ap. J. (Letters), $309, \mathrm{~L} 47$.

Walker, C. K., Lada, C. J., Young, E. T., and Margulis, M. 1988, Ap. J., 332, 335.

Whittet, D. C. B. 1974, Mon. Not. Roy. Astr. Soc., 168, 371.

Wilking, B. A. 1990, Low Mass Star Formation in Southern Molecular Clouds, in press.

Wilking, B. A. and Lada, C. J. 1983, Ap.J., 274, 698.

Wilking, B. A., Lada, C. J. and Young, E. T. 1989, Ap. J., 340, 823.

Wilking, B. A., Schwartz, R. D., and Blackwell, J. H. 1987, Astron. J., 94, 106.

Wootten A. 1989, $A p$. J., 337, 858.

Wootten, A., and Loren, R. B. 1987, Ap. J., 317, 220.

Wouterloot, J. G. A., and Habing, H. J. 1985, Astr. Ap. Suppl, 60, 43.

Wouterloot, J. G. A., Walmsley, C. M., and Henkel, C. 1988, Astr. Ap, 191, 323.

Wynn-Williams, C. G. 1982, Ann. Rev. Astr. Ap., 20, 597.

Young, E. T., Lada, C. J., and Wilking, B. A. 1986, Ap. J., 340, 823.

Zhou, S., Evans, N. J. II, Butner, H. M., Kutner, M. L., Leung, C. M., and Mundy, L. G. 1990a, Ap.J., 363, 168.

Zhou, S., Evans, N. J., II, and Mundy, L. G. 1990, Ap. J., 355, 159.

Zhou, S., Evans, N. J., II, Mundy, L. G., Güsten, R., and Kutner, M. L. 1991, $A p$. $J .$, submitted.

Zhou, S., Wu, Y., Evans, N. J., II, Fuller, G. A., and Myers, P. C. 1989, Ap. J., 346, 168. 